\title{
Orbit Paraganglioma
}

National Cancer Institute

\section{Source}

National Cancer Institute. Orbit Paraganglioma. NCI Thesaurus. Code C6408.

A benign or malignant extra-adrenal parag ang lioma arising from the orbit. Patients may present with visual disturbances or proptosis. 